\author{
Military Technical College \\ Kobry El-Kobbah, \\ Cairo, Egypt
}

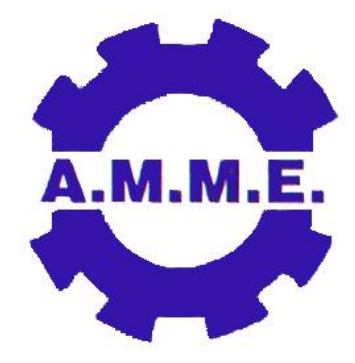

$14^{\text {th }}$ International Conference on Applied Mechanics and Mechanical Engineering.

\title{
Measurement Techniques and Characterization of High Speed Moving Objects
}

\author{
By \\ Matteo Lancini* $\quad$ Cinzia Amici** Claudio Breda**
}

\begin{abstract}
:
Response to fast speed stimuli of non linear system is difficult to predict without a proper model whose parameter has been characterized in condition closer to the working ones, because assumptions and simplifications on component stiffness and damping, such as their linearity in the frequency domain, are no longer valid.

To characterize a two degrees of freedom spring-mass-damper system reacting to a high speed impact a set of statistical techniques has been developed and applied to a set of experimental measurements obtained thanks to a high speed camera, a piezoelectric load cell and a set of piezoaccelerometers.
\end{abstract}

Photogrammetric position accuracy has been improved using a least square estimator of a single position from different markers, therefore position and acceleration data have been fused together being weighted on the reciprocal of their uncertainties.

Starting from load, position, velocity and acceleration data, spring-mass-damper systems with different degrees of freedom have been characterized in different configurations using genetic algorithms to minimize the square deviation of prediction from measured speed, although the term to be minimized has been again weighted on reverse of uncertainty to avoid false results.

Eventually the whole process has been validated and automated in order to provide a new and efficient mean of characterizing dynamic parameters such as stiffness, damping, trigger length of elastic systems impacted by high speed ${ }^{1}$ objects.

\section{Keywords:}


Proceeding of the $14^{\text {th }}$ AMME Conference, $25-27$ May 2010

sensor fusion, accelerometer, load cell, high speed camera, genetic algorithms, uncertainty, least square

* Assistant Professor - University of Brescia, Brescia, Italy

** PhD Candidate - University of Brescia, Brescia, Italy

*** Test Manager 


\section{Introduction:}

Identification of linear dynamic systems is nowadays a well known and investigated research field [1-5], although often and often the response predictions, offered by linear models, are not considered accurate enough. To improve them, also nonlinear physical phenomena need to be taken into account; this is one of the reasons why nonlinear dynamic problems are receiving an always growing attention in literature [6-14].

The system analysis becomes more and more critical if the dynamic process involves also high speed moving elements: in fact, whether pure impact dynamics are a traditional trivial topic [15-19], the model parameters estimation requires as a result an accurate experimental and numerical investigation [20-21].

In this paper, the response of a nonlinear dynamic system to high speed stimuli will be considered, and the measurement techniques adopted to characterize the model parameters will be therefore described.

After a brief presentation of the physical system and of the measurement chain, the implemented data analysis techniques and parameters characterization will be illustrated, while the most salient aspects of this study and its potential application fields will be finally summarized in the conclusions.

\section{The Case Study:}

The case study that was used to develop the proposed methods regards the characterization of damping properties of a composite biological material, specifically the natural composition of bone structures and soft tissues in a living body, subject to an abrupt local compression. On one side the material displays a viscoelastic behavior with non-linear characteristics, while on the other the stimulus, to which the response is sought, could be normally associated with an impulse signal in strength, although the material stiffness is not constant during the impulse, hence standard impulsive analysis techniques could not be directly used. Due to the material characteristics (low static stiffness, high damping, layered composition) the direct measurements weren't focused on the direct target of the investigation, but on the rigid element transmitting the stimulus, whose position and interfaces could be easily monitored, thanks to the fact that no mechanical constrains affect the actuator during its working phase.

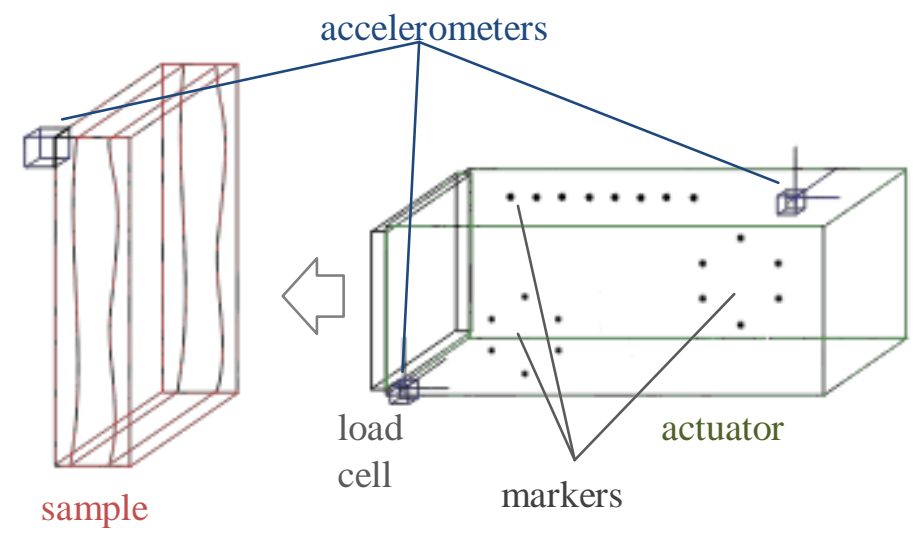


Figure (1): Mechanical model of material sample under test and actuator

The used instrumentation involves a series of seven uniaxial accelerometers (with a $500 \mathrm{kHz}$ cutoff frequency), a load cell integrated into the stimulus actuator (IEPE load cell), and a high speed camera. While data from the high speed camera was limited to a $20 \mathrm{kHz}$ sampling frequency, force and acceleration measurements were taken at $100 \mathrm{kHz}$ sampling frequency and then aligned to the photogrammetric data thanks to a trimmer signal (figure 1).

\section{Physical Model:}

A preliminary analysis has been performed, using a combination of proprietary software, excel, matlab and labview, on a set of force and longitudinal acceleration measurements to limit the models used for further investigations[7, 10]. Plotting the ratio of the force, measured at the interface, over acceleration -as shown in figure 2- a set of nearly constant values was noticed, hence the idea of simulating the material behavior using a model where stiffness changes abruptly between different states.

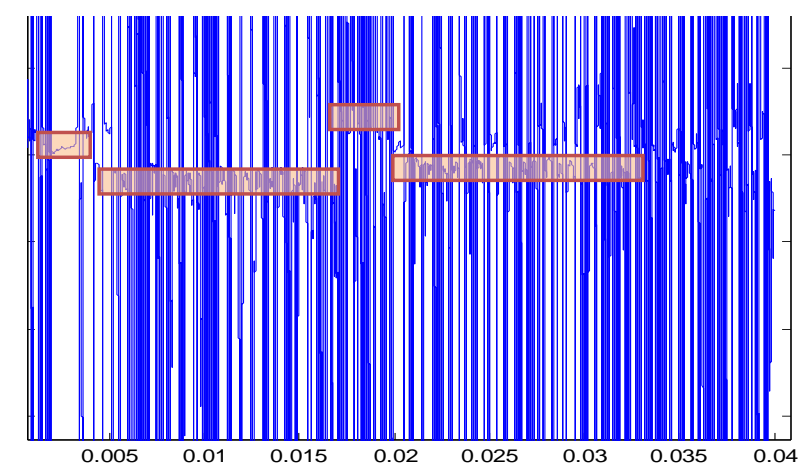

Figure (2): ratio of force on principal acceleration in time domain [s] ${ }^{*}$

Further preliminary tests regarded photogrammetric analysis: due to the availability of a single high speed camera, the best angle of perspective was sought in order to minimize distortions on displacement information.

The analysis of different takes of the same stimulus replicated on the same sample, pointed out angular and translational movements mainly related to two dimensions; therefore the model, used for simulation and interpretation of data, was simplified to be bidimensional, greatly increasing the video data source accuracy. Moreover, the deformations along the longitudinal axis (the actuator moving axis) was noticed to be predominant over the others, hence all analysis was focused on the principal deformation axis more than on the others.

These simplifications lead to the development of four different models for the simulation of the sample stiffness[16, 22-24]; starting from the simple spring-mass-damper with just one degree of freedom until the presented one, the model was improved thanks to a thorough analysis of the results:

1. Single degree of freedom with a linear spring-mass-damper model[25]

2. Single d.o.f. s-m-d model with a continuously variating stiffnes[11, 26-28]

3. Single d.o.f. s-m-d model with a stepped variating stiffnes[11, 26-28]

4. Two d.o.f. s-m-d model with a step relation between the dofs[29, 30] 
A first single degree of freedom model was the actual starting point, having synthesized the under study material with just two parameters: damping and stiffness; then its parameter were made variable with time. While the first model was an oversimplification unable to explain the actual behavior the second one was far too complex to be used into the solving algorithm needed for the characterization; the preliminary data suggested therefore the creation of different states for mass, stiffness and damping, actually two, switching the one in the other after a predetermined time delay.

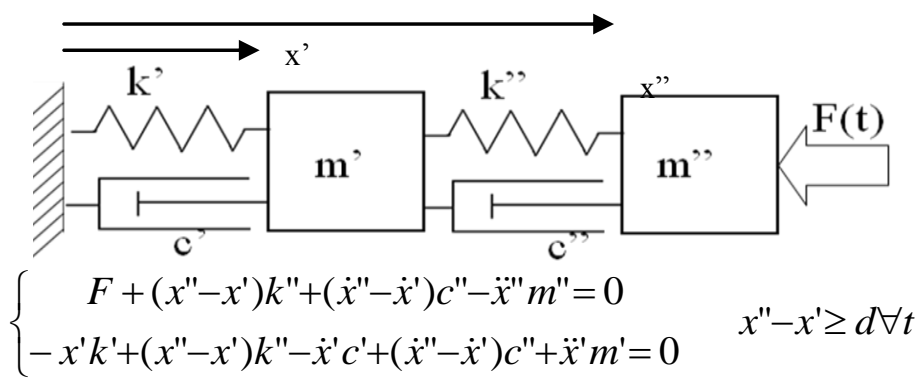

Figure (3): final physical mode $\left.\right|^{2}$

The first version of this model depended on a time delay between two different stiffness states: although the model presented good results, in the first experiments a constant time delay resulted unstable, therefore an improvement has been implemented, leading to the final model definition, where the switch between two different states depends on the actual travel distance of the actuator.

The actual model, displayed in figure 3 , synthesizes material behavior in 7 different parameters, which were estimated thanks to the genetic algorithms:

- Mass of the first element ${ }^{3}$

- Stiffness of the first connection

- Damping of the first connection

- Mass of the second element

- Stiffness of the second connection

- Damping of the second connection

- Travel distance of the linking switch

$\bullet$

\section{Preliminary Data Treatment:}

Due to the diversity of the acquired data, their sampling frequency and their different origin, a preliminary data treatment has been devised in order for them to be coherent and comparable, moreover, the data needed to be fit for a numeric simulation. This preliminary adaptation has been performed by combining different algorithms and techniques, both in order to reduce uncertainty and coordinate data.

Photogrammetric data is often subjected to high uncertainty due to light variation, camera resolution and marker dimensions: to increase accuracy of displacement data coming from the 
video feed a set of redundant marker has been located on the actuator, following two sets of six points in circles and one set of eight points in line.

Using the markers disposition a least square estimator has been used to identify the patter relevant points (center for circles and generator for line pattern): each marker trajectory has been estimated thanks to photogrammetric data, then for each set of markers a relevant point coordinates are extracted by minimizing the distance between the actual trajectories and the computed one.

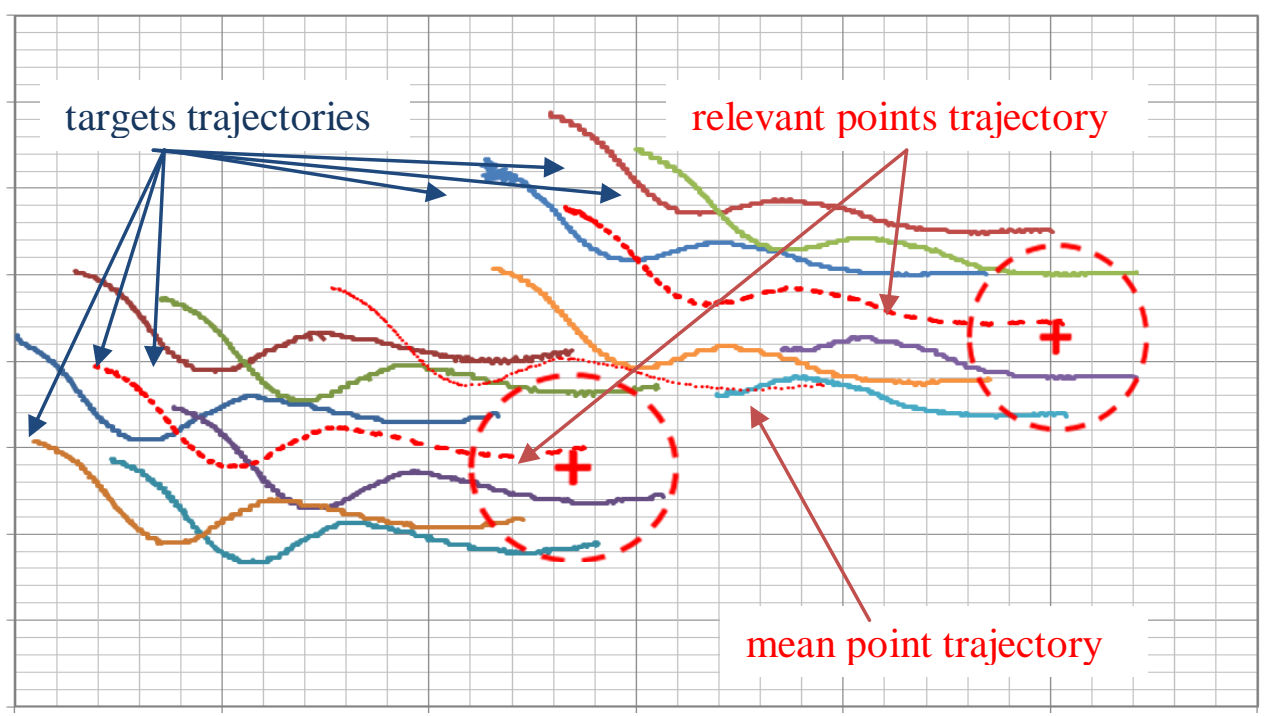

Figure (4): photogrammetric data treatment results

This trajectories reconstruction showed a very good result (figure 4), having a $\mathrm{R}^{2}$ indicator (the fraction of variance explained by the model) never below $99.95 \%$, and reducing resolution based uncertainty to one third, moreover, the usage of redundant markers on a rigid element, where two points coordinates would suffice, allows to keep tracking the element trajectories also when occasional loss of track of one or more markers do happen.

After extracting the central point trajectory data and the actuator angle history, all data needed to be translated and rotated in order to be compatible with actuator accelerations and load data: photogrammetric data is in fact recorded in a fixed, absolute reference system, while load and IEPE data came from sensor directly attached to the actuator, therefore subjected to the same rotations and translations, hence all data is referred to a moving reference system linked to the actuator. On the other side, a vectorial composition was required for extracting from accelerometers data information about angular acceleration: a vector describing the relative displacement of one accelerometer with respect to the other has been used, thanks the fact that all acceleration data triplets were punctual, orthogonal and aligned with the other triplet. 


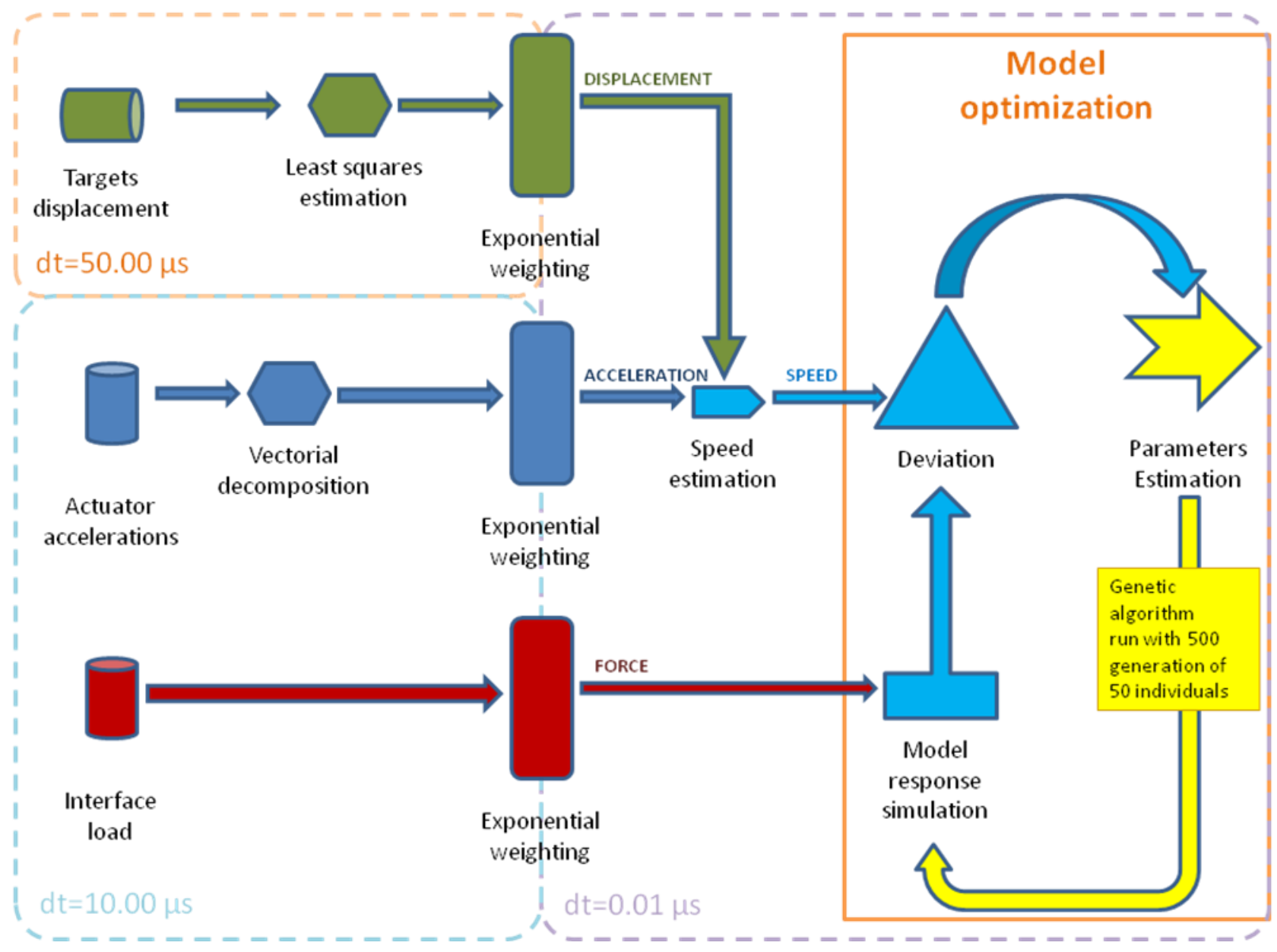

Figure (5): data post processing treatment sequence

While acceleration was measured and displacement estimated thanks to photogrammetric data, speed was computed as a composition of the latter two sources: acceleration was integrated to obtain an estimate of speed, and then corrected by averaging it with the first derivative of displacement.

All data until now gathered up, has further to be adapted to numerical simulation: specifically, in order for a Runke-Kutta integration to work, the timing interval between the samples has to be reduced to $0.1 \mu \mathrm{s}$, and the signals have to share the same timebase for the simulations to work. To recreate a signal with a given timebase starting from measurement with another timebase, an exponential weighting time-based filter was used, approximating the value of a signal at a given time with the average of historical values of the same property weighted on the exponential time difference between the instant being simulated and the data being weighted.

Using the actuator velocity as a target and the load at the interface as input the proposed model was characterized, optimizing the presented seven parameters thanks to a genetic algorithm with 500 generation of 50 individuals: for each individual a Runge-Kutta simulation [31] was used to calculate the longitudinal speed of the actuator, hence the square mean deviation of the actual speed from the simulated one as the term to be minimized by the genetic algorithms. 


\section{Conclusions:}

Although the used model was quite simple with respect to the complex material under examination, results showed a good correspondence between the optimized model and the simulated one, displaying a very low deviation, not only for comparison in terms of speed, but also in terms of acceleration and displacement, as could be seen in figures 6 and 7 .

The case study offered the chance to validate the proposed methods showing how they can be successfully used to characterize models for high speed moving objects; in particular it has been displayed how a set of techniques could be combined to enhance accuracy and reliability of model characterization when fast reactions are used as input or outputs of the model under examination.

Although only a single result is hereby displayed, 20 tests were taken investigating the robustness of the approach, especially repeating the same test in different moments and with different maximum load values: all tests showed a very low deviation of predicted cinematic parameters from the measured ones, even if the natural variability of biological material lead to variation in both stimulus and response.

Further developments of these techniques will be their application to a wider set of experimental cases in different conditions, their combination with more complex models, and the creation of composite software which bundles the various techniques, here presented together, in order to avoid the need for manual coordination between the different software and devices which actually make the proposed combined techniques slow and unsuitable for a test or production usage.

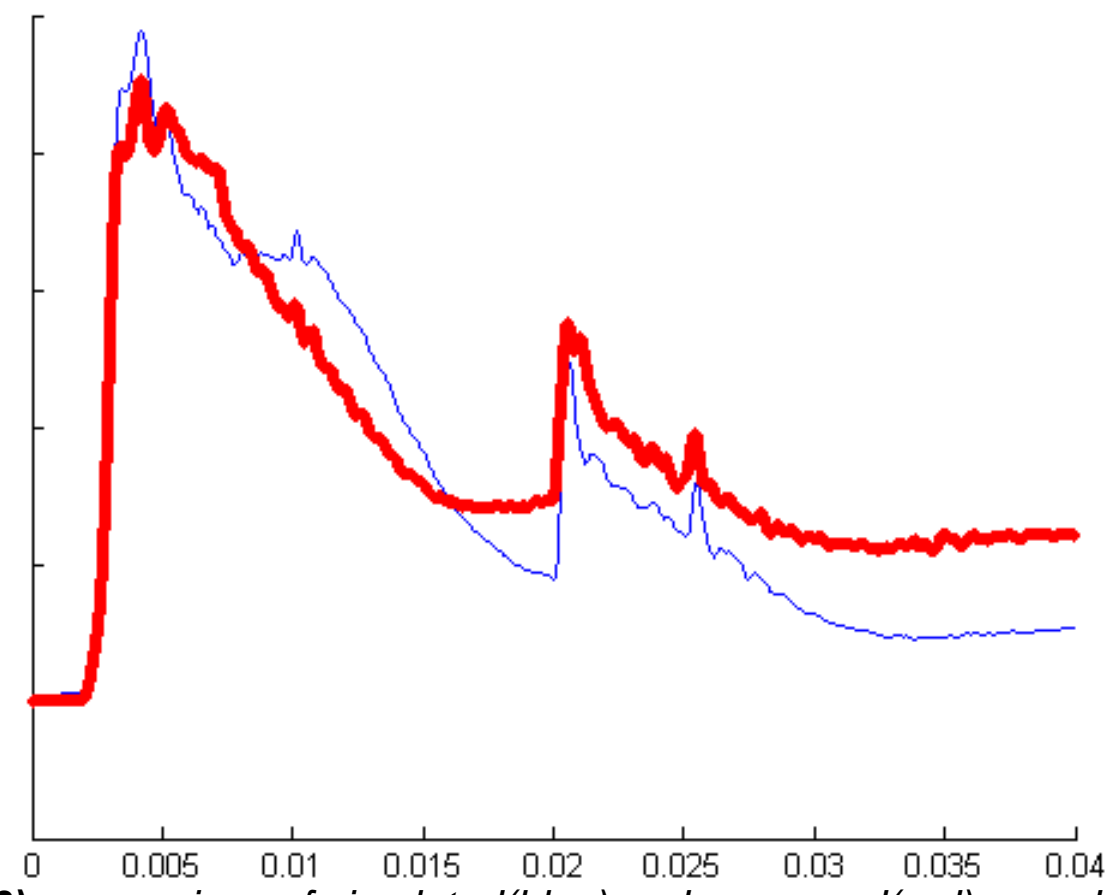

Figure (6): comparison of simulated(blue) and measured(red) speed in time [s] ${ }^{\star}$ 


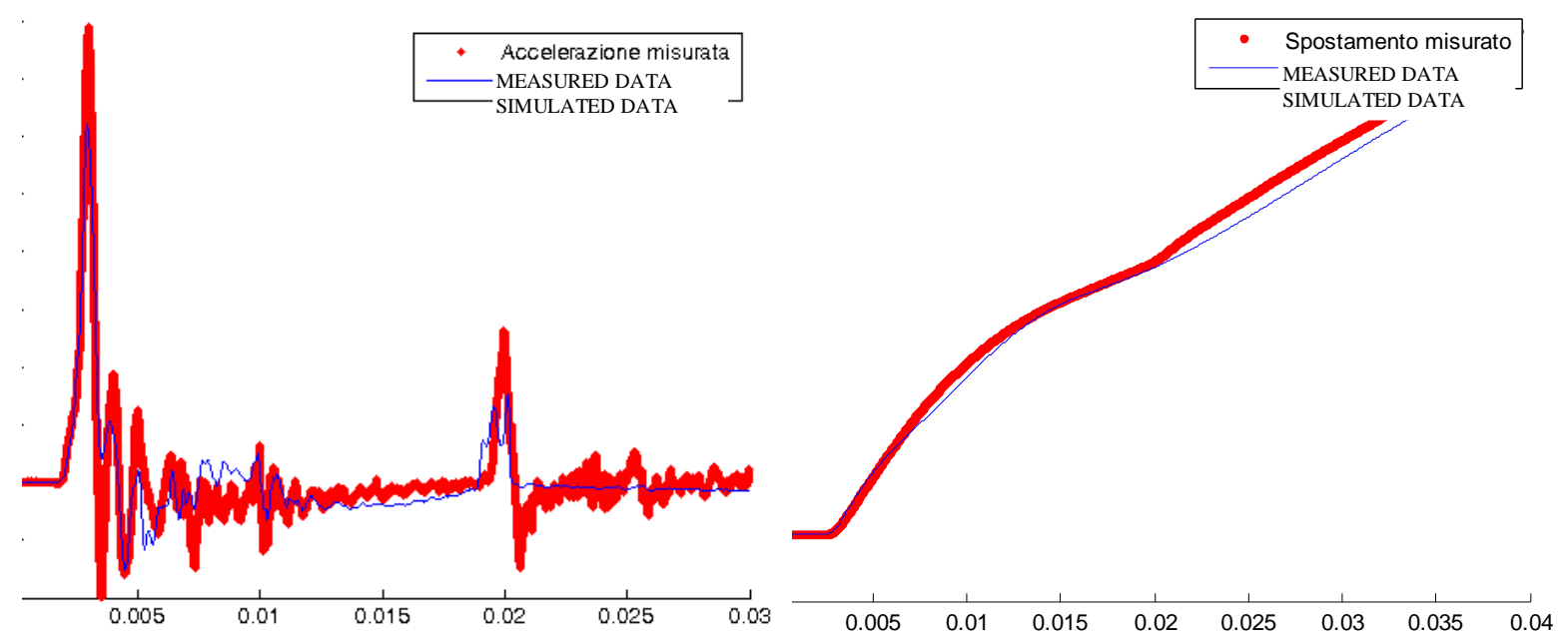

Figure (7): comparison of acceleration (on left) and displacement (right) in time[s] $]^{*}$

\section{References:}

1. H. G. D. Goyder, "Methods and application of structural modelling from measured structural frequency response data", Journal of Sound and Vibration, Vol 68, pp. 209230, 1980.

2. R. Potter and M. Richardson, "Mass stiffness and damping from measured modal analysis data", ISA Paper No. 74-630,1-I 5. 1974.

3. K. Shve and M. Richardson, "Mass, stiffness and damping matrix estimates from structural measurements", Proceedings of the 5th International Modal Analysis Conference, London, England, pp. 756-761. 1987.

4. J. Leuridan, "Some direct parameter model identification methods applicable for multiple input modal analysis", Ph.D. Dissertation, Department of Mechanical and Industrial Engineering, University of Cincinnati, Cincinnati, U.S.A, 1984.

5. F. Lembregts, J. Leuridan and H. V. Brussel, "Frequency domain direct parameter identification for modal analysis: state space formulation", Proceedings of the 13th International Seminar on Modal Analysis, Katholieke University, Leuven, Session 6C, 54. 1988.

6. K. S. Mohammad, K. Worden, G. R. Tomlinson, "Direct parameter estimation for linear and non-linear structures", Journal of Sound and Vibration, Vol. 152 (3), pp. 471-499, 1992

7. H. J. Rice, J. A. Fitzpatrick, "A procedure for the identification of linear and non-linear multi-degree-of-freedom systems", Journal of Sound and Vibration, Vol. 149 (3), pp. 397-411, 1991.

8. P. A. Atkins, J. R. Wright, K. Worden, "An extension of force appropriation to the identification of non-linear multi-degree of freedom systems", Journal of Sound and Vibration, Vol. 237 (1), pp. 23-43, 2000.

9. A. H. Nayfeh, "Random motion and dynamic response: Parametric identification of nonlinear dynamic systems" Computer \& Structures, Vol. 20, pp. 487-493, 1985.

10.S. Setio, H. D. Setio, L. Jezequel, "A method of non-linear modal identification from frequency response tests", Journal of Sound and Vibration, Vol. 158 (3), pp. 497-515, 1992. 
11.S. M. Spottswood, R. J. Allemang, "Identification of nonlinear parameters for reduced order models", Journal of Sound and Vibration, Vol. 295, pp. 226-245, 2006.

12. S. F. Masri, H. Sassi and T. K. Caughey, "A nonparametric identification technique for nonlinear dynamic problems" ASME Journal of Applied Mechanics, Vol.49, pp. 433447, 1979.

13. S. F. Masri, H. Sassi and T. K. Caughey, "Nonparametric identification of nearly arbitrary nonlinear systems", ASME Journal of Applied Mechanics, Vol.49, pp. 619628, 1982.

14. Y. Yang and S. R. Ibrahim, "A nonparametric identification technique for a variety of discrete nonlinear vibrating systems", ASME Journal of Vibration, Acoustics, Stress, and Reliability in Design, Vol. 107, pp. 60-66, 1985.

15. S. Heimbs, S. Heller, P. Middendorf, F. Hähnel, J. Weiße, "Low velocity impact on CFRP plates with compressive preload: Test and modelling", Int. J. Impact Engng, Vol. 36, pp. 1182-1193, 2009.

16.D.D. Quinn, K. Bairavarasu, "Near-simultaneous impacts", Int. J. Impact Engng, Vol. 32, pp. 889-904, 2006.

17. A. P. Kren, A. O. Naumov, "Determination of the relaxion function for viscoelastic materials at low velocity impact", Int. J. Impact Engng, Vol. 37, pp. 170-176, 2010.

18. S. Pashah, M. Massenzio, E. Jacquelin, "Prediction of structural response for low velocity impact", Int. J. Impact Engng, Vol. 35, pp. 119-132, 2008.

19. N. C. Menh, "Responses of weakly non-linear dynamical systems subjected to random parametric and external excitations", Journal of Sound and Vibration, Vol. 113 (1), pp. 1-8, 1987.

20. M. Di Sciuva, C. Frola, S. Salvano, "Low and high velocity impact on Inconel 718 casting plates:ballistic limit and numerical correlation", Int. J. Impact Engng, Vol. 28, pp. 849-876, 2003.

21. R. R. V. Neves, G. B. Micheli, M. Alves, "An experimental and numerical investigation on tyre impatc", Int. J. Impact Engng, Article in press, 2009.

22. K. Q. Wu, T. X. Yu, "Simple dynamic models of elastic-plastic structures under impact", Int. J. Impact Engng, Vol. 25, pp. 735-754, 2001.

23. S. C. Sinha, M. Jagannathan, "Pulse response of non-linear non-stationary vibrational systems with N degrees of freedom", Journal of Sound and Vibration, Vol. 112 (2), pp. 237-248, 1987.

24. D. J. Olbering, S. C. Sinha, "Response of non-linear non-stationary systems subjected to pulse excitation", Journal of Sound and Vibration, Vol. 112 (2), pp. 227-235, 1987.

25.C. Fritzen, "Identification of mass, damping and stiffness matrices of mechanical systems", ASME Journal of Vibration, Acoustics, Stress, and Reliability in Design Vol 108, pp. 9-16. 1986.

26. E. Barkanov, W. Hufenbach, L. Kroll, "Transient response analysis of systems with different damping models", Comput. Methods Appl. Mech. Engrg, Vol. 192, pp. 33-46, 2003

27. R. Vaziri, X. Quan, M. D. Olson, "Impact analysis of laminated composite plates and shells by super finite elements", Int. J. Impact Engng, Vol. 18, pp. 765-782, 1996.

28. V. P. W. Shim, K. Y. Yap, "Modelling impact deformation of foam-plate sandwich systems", Int. J. Impact Engng, Vol. 18, pp. 615-636, 1997.

29. G. Bolzen, R. Fedele, G. Maier, "Parameter identification of a cohesive crack model by Kalman filter", Comput. Methods Appl. Mech. Engrg, Vol. 191, pp. 2847-2871, 2002 
30.F. Ma, A. Imam, M. Morzfeld, "The decoupling of damped linear systems in oscillatory free vibration", Journal of Sound and Vibration, Vol. 324, pp. 408-428, 2009.

31. John C. Butcher (2003). Numerical methods for ordinary differential equations. John Wiley \& Sons. ISBN 0471967580

\section{Notes:}

1 The speed, as well as the specific application of this technique, is a trade confidential information and is therefore undisclosed to the public.

${ }^{2}$ All figures are reported without scales or unit of measurement due to a non disclosure agreement: only the time scale is reported to present the method, actual results are a trade confidential information.

${ }^{3}$ The mass found thanks to the genetic algorithm has been found to be compatible (less than $5 \%$ deviation) with the actual moving mass of the application, which ranged between $1 \mathrm{~kg}$ and $5 \mathrm{~kg}$ depending on the test undertaken. 